\title{
Necrose esofágica aguda ("Black Esophagus") em paciente portador de neoplasia neuroendócrina do pâncreas: relato de caso
}

\author{
Acute esophagic necrosis ("Black Esophagus") in patient with neuroendocrine neoplasia of \\ pancreas: case report
}

\author{
Carolina Pontes de Moraes Hungria ${ }^{1}$, Isabella Moreira Hueb ${ }^{1}$, Marina Santos Noia ${ }^{1}$, Lilian Nomura da \\ Silva ${ }^{1}$, Tiago Bezerra de Freitas Diniz ${ }^{2}$, Danilo Gagliardi ${ }^{3}$
}

\section{Resumo}

A Necrose Esofágica Aguda (NEA) é uma síndrome rara, caracterizada pela presença de pigmentação escura difusa no esôfago observada por endoscopia. O diagnóstico é frequentemente incidental em pacientes com sintomas de sangramento gastrointestinal superior. Neste artigo apresenta-se o caso de um paciente do sexo masculino, de 72 anos, encaminhado ao Departamento de Cirurgia da Irmandade da Santa Casa de Misericórdia de São Paulo (ISCMSP) com história de dor epigástrica persistente e úlceras gástricas refratárias ao tratamento clínico. Foi realizada Endoscopia Digestiva Alta (EDA) de controle que visualizou lesão no bulbo duodenal, cuja biópsia e imunohistoquímica revelaram tumor carcinoide e neuroendócrino. Paciente foi submetido à cirurgia de enucleação de tumor na cabeça do pâncreas, evoluindo com fístula pancreática no sétimo dia de pós operatório. No décimo dia de pós-operatório, retornou ao Pronto Socorro do Serviço de Emergência com queixa de dor abdominal intensa, fezes escurecidas e presença de melena ao toque retal. A EDA diagnosticou necrose esofágica aguda. Após a identificação da NEA, foi introduzido antibioticoterapia de amplo espectro (Piperacilina + Tazobactan), sintomáticos e vigilância infecciosa, evoluindo bem ao tratamento conservador.

Descritores: Necrose, Esôfago/patologia, Doença aguda,

1. Acadêmico da Faculdade de Ciências Médicas da Santa Casa de São Paulo - $6^{\circ}$ Ano do Curso de Graduação em Medicina

2. Médico Residente Irmandade da Santa Casa de Misericórdia de São Paulo-Departamento de Cirurgia

3. Professor Adjunto da Faculdade de Ciências Médicas da Santa Casa de São Paulo - Departamento de Cirurgia

Trabalho realizado: Irmandade da Santa Casa de São Paulo. Departamento de Cirurgia

Endereço para correspondência: Carolina Pontes de Moraes Hungria. Rua Doutor Albuquerque Lins, 1169 Apto 92 A - Santa Cecília - 01230-001 - São Paulo - SP - Brasil. E-mail: carolinaponteshungria@gmail.com
Esofagite, Tumores neuroendócrinos, Pâncreas, Endoscopia do sistema digestório

\begin{abstract}
Acute Esophageal Necrosis (NEA) is a rare syndrome, characterized by the presence of diffuse dark pigmentation in the esophagus observed by endoscopy. The diagnosis is often incidental in patients with symptoms of upper gastrointestinal bleeding who are submitted to upper gastrointestinal endoscopy. This article presents a case referred to the Surgery Department of the Santa Casa de Misericórdia de São Paulo (ISCMSP) of a 72-year-old male patient with a history of persistent epigastric pain and gastric ulcers refractory to clinical treatment. High Digestive Endoscopy (EDA) was performed, which showed lesion in duodenal bulb, biopsy and immunohistochemistry with results of carcinoid tumor and neuroendocrine tumor. Patient underwent enucleation surgery of the pancreas head and evolved with pancreatic fistula seven days after surgery. On the tenth postoperative day, the patient returned to the Emergency Room with complaints of severe abdominal pain, darkened stools and the presence of melena at the rectal touch, requiring EDA to diagnose acute esophageal necrosis. After identification of the NEA, broad-spectrum antibiotic therapy (Piperacillin + Tazobactan), symptomatic and infectious surveillance, were successfully developed, with conservative treatment,
\end{abstract}

Keywords: Necrosis; Esophagus/pathology; Acute disease; Esophagitis; Neuroendocrine tumors; Pancreas; Endoscopy, digestive system

\section{Introdução}

A Necrose Esofágica Aguda (NEA), também conhecida como "Black Esophagus" ou Esofagite Necrotizante, é uma síndrome rara, descrita pela primeira vez em 1990, por Goldenberg ${ }^{(1)}$. Caracteriza-se pela presença de pigmentação escura difusa no esôfago e 
ulcerações, correspondendo à inflamação aguda grave e necrose da mucosa ${ }^{(2)}$. Este trabalho tem por objetivo relatar o caso de um paciente atendido na Santa Casa de São Paulo que evoluiu com necrose esofágica aguda após internação para ressecção cirúrgica de neoplasia neuroendócrina do pâncreas.

\section{Relato de Caso}

Paciente do sexo masculino, 72 anos, em acompanhamento há cinco anos por dor epigástrica persistente e úlceras gástricas refratárias ao tratamento clínico no ambulatório de gastroenterologia clínica da Santa Casa de São Paulo. Referia como antecedentes pessoais: ressecção transuretral de próstata por HPB há dez anos, hernioplastia inguinal bilateral há oito anos, ressecção de pterígio há nove meses, ex-tabagismo (10 anos/ maço) e tratamento de H. pylori em 2013.

Durante EDA controle, foi visualizada lesão elevada no bulbo duodenal, cuja biópsia e imunohistoquímica revelaram tumor carcinoide e neuroendócrino de baixo grau, respectivamente. Foi solicitada tomografia do abdômen que mostrou formação expansiva, infiltrativa e hipervascularizada, com origem presumida na segunda porção duodenal e com extensão para a cabeça do pâncreas. A principal hipótese diagnóstica considerada foi neoplasia neuroendócrina. Paciente foi então encaminhado para o Departamento de Cirurgia.

Em consulta inicial no ambulatório de cirurgia, apresentava-se em bom estado geral, corado, hidratado, anictérico, acianótico, afebril, lúcido, sem alterações cardiovasculares ou respiratórias, com abdômen plano, flácido, indolor a palpação, sem massas ou visceromegalias.Após discussão do caso pela equipe de Cirurgia do Pâncreas e Vias Biliares, foi indicada a realização de gastroduodenopancreatectomia (Cirurgia de Whipple).

Durante a cirurgia, constatou-se tumor pediculado localizado na cabeça do pâncreas com plano de clivagem com o duodeno. Foi então optada pela enucleação da lesão e sutura de sua base em dois planos, seguida de drenagem da cavidade próxima ao leito da ressecção. Paciente evoluiu com fístula pancreática tipo A de baixo débito (amilase de dreno de 48h: 1356 u/l) e no sétimo dia de pós operatório, estável hemodinamicamente, em bom estado geral e assintomático, recebeu alta hospitalar em uso do dreno com seguimento ambulatorial.

Três dias após a alta (décimo dia pós-operatório), retornou ao Pronto Socorro do Serviço de Emergência da ISCMSP com queixa de dor abdominal intensa e fezes escurecidas. Ao exame físico, apresentava-se hemodinamicamente normal, com dor epigástrica a palpação e sem sinais de peritonite. Havia melena no toque retal. Diante do quadro, foram solicitados exames laboratoriais e nova endoscopia.

\begin{tabular}{lcc}
\hline EXAME & RESULTADO & $\begin{array}{c}\text { VALOR } \\
\text { REFERÊNCIA }\end{array}$ \\
\hline Amilase dreno & 19336 & \\
\hline Amilase sérica & 3736 & $<220$ \\
\hline
\end{tabular}

A endoscopia evidenciou esôfago livre em sua luz, recoberto por mucosa íntegra e de coloração normal até $24 \mathrm{~cm}$ da arcada dentária superior, a partir de onde constatou-se mucosa de coloração enegrecida circunferencialmente, com áreas de ulcerações recobertas por espessa camada de fibrina e necrose, que se estendia até transição esofagogástrica. Os achados endoscópicos sugeriam necrose esofágica aguda (Black Esophagus).

O paciente foi internado, mantido em jejum, introduzido antibioticoterapia de amplo espectro (Piperacilina + Tazobactan), sintomáticos e vigilância infecciosa. Durante internação, respondeu adequadamente ao tratamento conservador, evoluindo com alguns episódios de diarreia, com melena residual, afebril e estável hemodinamicamente. Após oito dias, foi solicitada nova
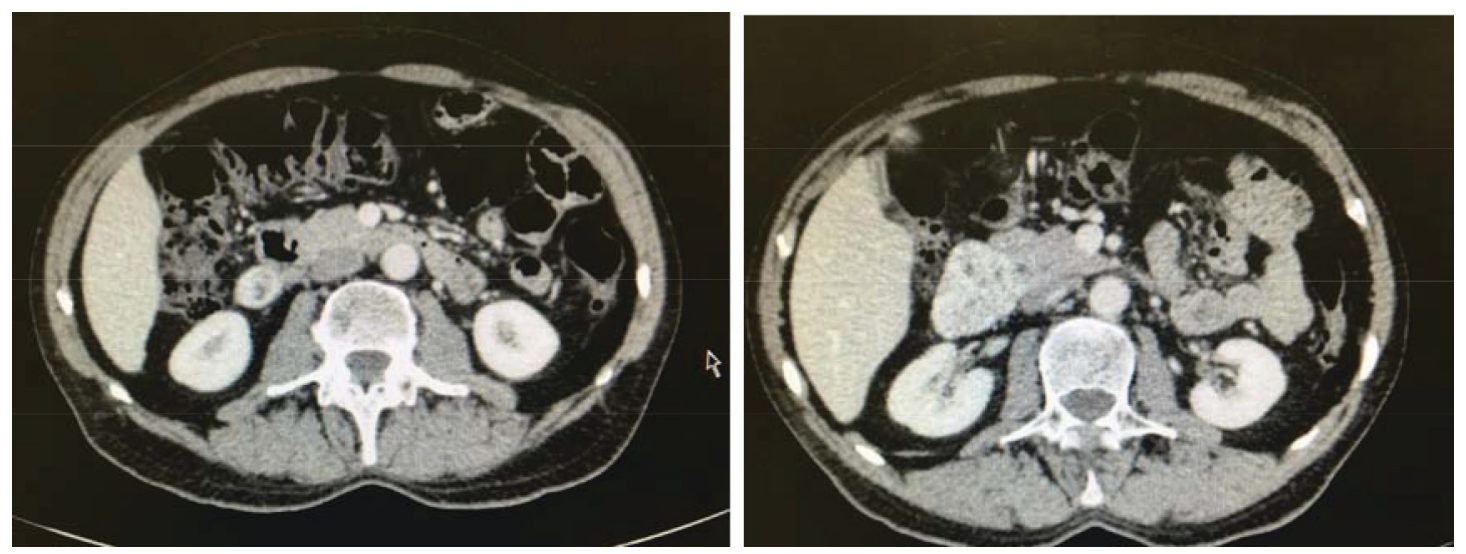

Figura 1 - Tomografia Computadorizada de abdômen exibindo a formação com origem presumida na segunda porção duodenal e com extensão para a cabeça do pâncreas. 


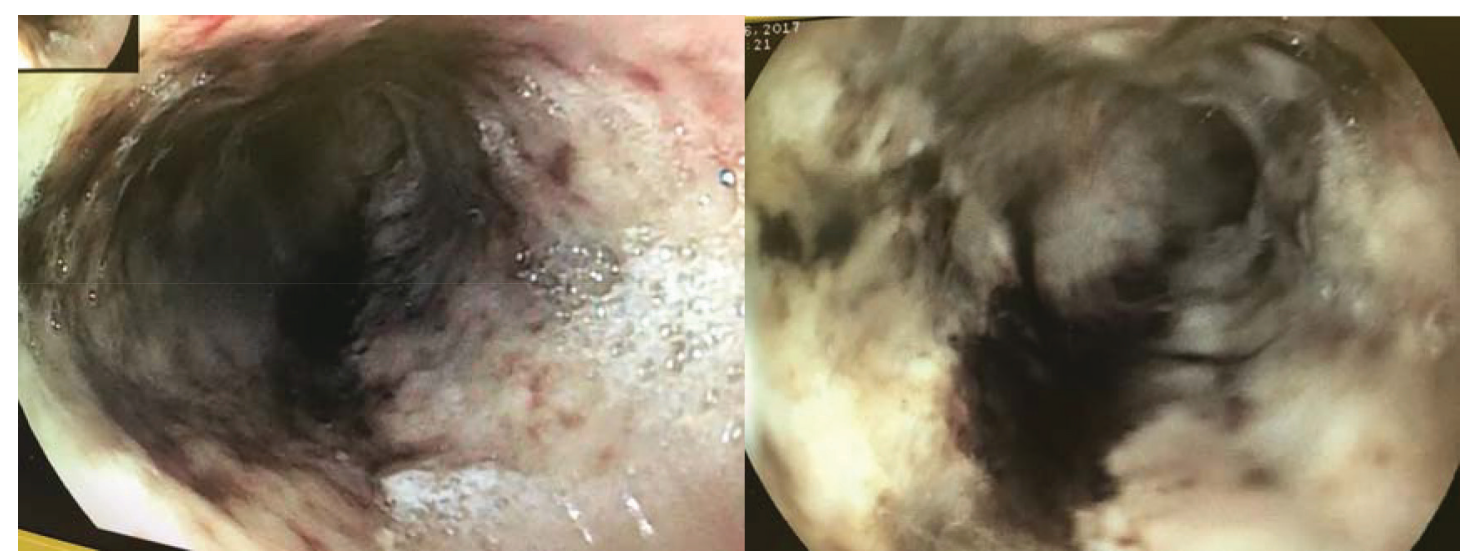

Figura 2 - Endoscopia Digestiva Alta evidenciando mucosa de coloração enegrecida circunferencialmente, com áreas de ulcerações recobertas por espessa camada de fibrina e necrose, sugerindo necrose esofágica aguda (Black Esophagus).

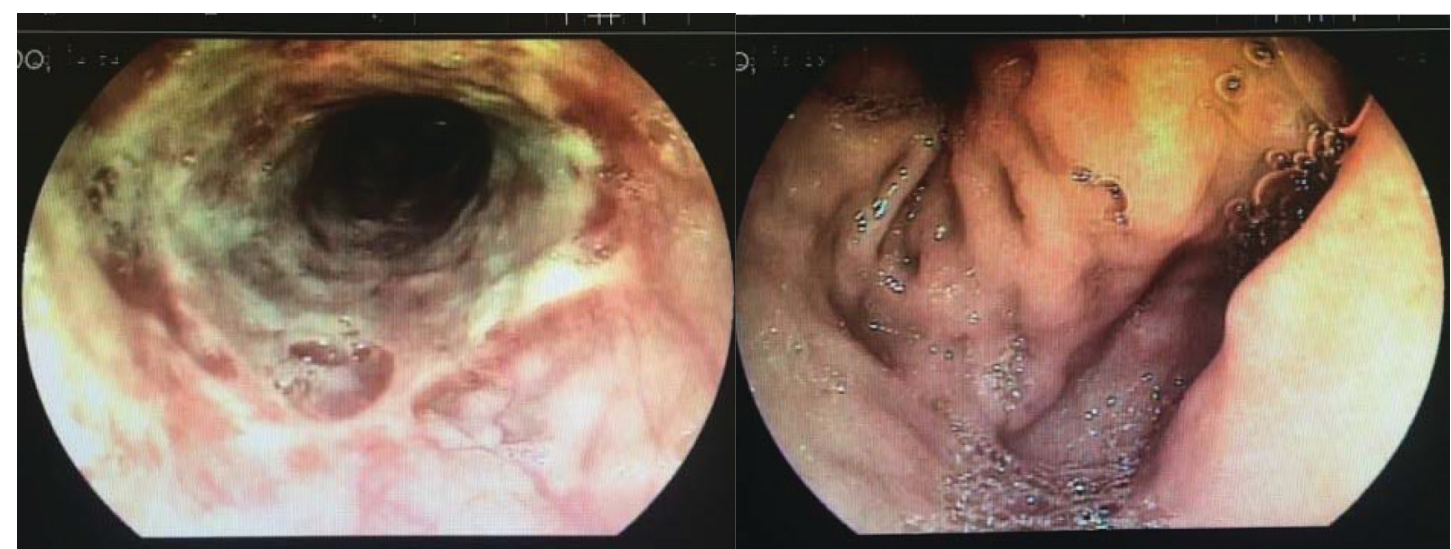

Figura 3 - EDA de controle com mucosa de coloração enantemática, recoberta por fibrina e com algumas erosões superficiais, mostrando melhora do quadro.

EDA de controle que mostrou melhora do quadro de necrose. A partir de $24 \mathrm{~cm}$ da arcada dentária (local da necrose) notou-se mucosa de coloração enantemática, recoberta por fibrina e com algumas erosões superficiais, que se estendiam até a junção esofagogástrica, sugerindo melhora do quadro de necrose.

Paciente evoluiu satisfatoriamente, recebeu alta vinte e três dias após cirurgia e treze dias após o retorno ao Pronto Socorro, sem dreno, HB 11,1 e Leucócitos de 5,7 mil sem desvio.

No retorno ambulatorial, quinze dias após alta, referiu boa aceitação da dieta e que se manteve assintomático. $\mathrm{O}$ resultado da imunohistoquímica mostrou imunoexpressão de gastrina, sinaptosina, E-caderina e KI67 < 1\%, sendo compatíveis com tumor neuroendócrino grau histológico 1, bem diferenciado, do pâncreas.

\section{DISCUSSÃO}

\section{Epidemiologia e Etiologia}

Segundo dados da literatura, estima-se uma preva- lência de até 0,2 por cento em autópsias e de 0,001 a 0,2 por cento em achados de endoscopia ${ }^{(3-4)}$. A incidência de NEA é cerca de quatro vezes maior em homens do que em mulheres e os pacientes têm em média 68 anos de idade ao diagnóstico ${ }^{(5)}$.

A etiologia não é bem definida, mas a isquemia parece ser o principal mecanismo, uma vez que o acometimento do esôfago, na maioria dos casos, é distal, onde o órgão é menos vascularizado ${ }^{(6)}$. Em relatos de caso, é comum a associação ao uso de antibióticos de amplo espectro, infecções (por Candida albicans, Citomegalovírus, vírus do herpes e Klebsiella pneumoniae), hérnia paraesofágica, cetoacidose diabética, síndrome de Stevens-Johnnyson, vômitos prolongados, hepatite alcoólica, acidose lática, dissecção aórtica, e neoplasias ${ }^{(7)}$ O doente em questão tem como condição que precede a NEA a presença de doença maligna subjacente.

\section{Quadro Clínico}

Em $70 \%$ dos casos, a Necrose Esofágica Aguda manifesta-se como hemorragia gastrointestinal 
Hungria COM, Hueb IM, Noia MS, Silva LN, Diniz TBF, Gagliardi D. Necrose esofágica aguda (“Black Esophagus”) em paciente portador de neoplasia neuroendócrina do pâncreas: relato de caso. Arq Med Hosp Fac Cienc Med Santa Casa São Paulo. 2018;63(2):126-30.

superior, com presença de hematêmese e melena ${ }^{(8)}$, além de outros sintomas gastrointestinais tais como: disfagia, dor epigástrica e dor torácica. Os sintomas relacionados à doença subjacente e sinais de sepse, como taquicardia e hipotensão, são possíveis também.

\section{Diagnóstico}

Devido aos sintomas referidos, o paciente é levado a realizar uma Endoscopia Digestiva Alta ${ }^{(9)}$ (como no caso relatado). Nesse exame foi revelada a presença de pigmentação escura difusa no esôfago, com ulcerações, correspondendo à inflamação aguda grave e necrose da mucosa. A NEA pode ser reconhecida pelas seguintes características: esôfago negro circunferencial, com ou sem exsudados; compromisso distal com extensão proximal; linha bem demarcada entre as lesões esofágicas e a mucosa gástrica, que se apresenta sem alterações ${ }^{(7)}$.

Os achados laboratoriais não são específicos de Necrose Esofágica Aguda e muitas vezes são devidos à doença de base. Eles incluem: acidose lática, hipoalbuminemia, anemia, insuficiência renal e hiperglicemia.

\section{Tratamento}

O tratamento da NAE não é específico, devendo ser manejado de forma análoga a condições clínico-patológicas semelhantes. Do ponto de vista local, justifica-se a administração endovenosa de inibidores da secreção ácida (antagonistas H2 ou inibidores da bomba de prótons). As medidas gerais incluem hidratação adequada, tratamento da doença de base e nutrição parenteral temporária ${ }^{(10)}$.

Os antibióticos empíricos devem ser iniciados em casos de suspeita de perfuração esofágica, descompensação clínica rápida, febres inexplicadas e comprometimento imune em AIDS, cirrose, receptor de transplante e pacientes em diálise. $\mathrm{O}$ uso profilático de antibióticos na necrose estéril não é, provavelmente, necessário. Existem poucos relatos correlacionando o uso de antibiótico com o desenvolvimento de $\mathrm{NEA}^{(11)}$.

A intervenção cirúrgica em pacientes com NEA é reservada para o esôfago perfurado, o qual resulta em mediastinite e formação de abscessos. Esofagectomia, decorticação, lavagem, e a reconstrução tardia podem ser realizadas na presença de achados intra-operatórios, de necrose esofágica com perfuração, volvo gástrico ou lesão aórtica.

A esofagectomia subtotal e a esofagogastrostomia foram relatadas na estenose esofágica induzida por NEA a qual era refratária a dilatações repetidas ${ }^{(1,12)}$.

\section{Complicações e Prognóstico}

A perfuração esofágica é uma complicação aguda da NEA que ocorre em menos de $7 \%$ dos pacientes, mas requer intervenção cirúrgica urgente. As estenoses esofágicas são complicações a longo prazo, e ocorrem em 25 a 40 por cento dos pacientes ${ }^{(13)}$. Os pacientes com estenoses esofágicas geralmente apresentam disfagia progressiva, com necessidade de dilatação por via endoscópica.

Com cuidados de suporte, a resolução dos achados endoscópicos ocorre na maioria dos pacientes ${ }^{(5)}$. No entanto, as taxas de mortalidade em pacientes com NEA variam de 13 a35 por cento ${ }^{(5,10,14)}$, associado principalmente a doença subjacente.

\section{CONCLUSÃO}

O tratamento conservador com antibioticoterapia profilática associado a vigilância para possíveis complicações associadas à necrose esofágica aguda mostrou-se uma conduta válida e eficaz no presente relato. Faz-se necessário um seguimento clínico com suporte de equipe cirúrgica e endoscopia para acompanhamento do caso.

A necrose esofágica aguda é uma complicação rara e deve fazer parte da gama de diagnósticos diferenciais do médico que se depara com quadro de hemorragia digestiva nos pacientes em pós-operatório recente.

\section{Referências}

1. Goldenberg SP, Wain SL, Marignani P. Acute necrotizing esophagitis. Gastroenterology. 1990; 98(2):493-6.

2. Castaño Llano R, Matar Khalil O, Cárdenas Vásquez A, Juliao Baños F, Sanín Fonnegra E, Erebrie Granados F. El esófago negro: ¿Patología infrecuente o desconocida? Rev Colomb. Gastroenterol. 2006; 21(1):62-7.

3. Etienne JP, Roge J, Delavierre P, Veyssier P. Nécroses de l'oesophage d'origine vasculaire. Sem Hop. 1969; 45(23):1599606.

4. Postlethwait RW, Musser AW; Changes in the esophagus in 1,000 autopsy specimens. J Thorac Cardiovasc Surg. 1974; 68(6):953-6.

5. Ben Soussan E, Savoye G, Hochain P, Hervé S, Antonietti M, Lemoine $\mathrm{F}$, et al. Acute esophageal necrosis: a 1-year prospective study. Gastrointest Endosc. 2002; 56(2):213-7.

6. Julián Gómez L, Barrio J, Atienza R, Fernández-Orcajo P, Mata L, Saracibar E, et al. Acute esophageal necrosis. An underdiagnosed disease. Rev Esp Enferm Dig. 2008;100(11):701-5.

7. Araújo R, Castanheira A, Machado J, Ramalho A, Eugénia Cancela, Ministro P, et al. Necrose esofágica aguda. Acta Med Port. 2011; 24(4):653-6

8. Gurvits GE, Shapsis A, Lau N, Gualtieri N, Robilotti JG. Acute esophageal necrosis: a rare syndrome. J Gastroenterol. 2007; 42(1):29-38.

9. Mishkin DS, Gelrud D. Acute esophageal necrosis (black esophagus). UpToDate. [on line]. Literature review current through: Jan 2018. This topic last updated: Feb 06, 2017. Available from: https:/ / www.uptodate.com/contents / acute-esophagealnecrosis-black-esophagus [2019 Jan 12] 
10. Lacy BE, Toor A, Bensen SP, Rothstein RI, Maheshwari Y. Acute esophageal necrosis: report of two cases and a review of the literature. Gastrointest Endosc. 1999; 9(4 Pt 1):527-32.

11. Mangan TF, Colley AT, Wytock DH. Antibiotic-associated acute necrotizing esophagitis. [Letter]. Gastroenterology. 1990; 99(3):900.

12. Kim YH, Choi SY. Black esophagus with concomitant candidiasis developed after diabetic ketoacidosis. World J Gastroenterol. 2007; 13(42):5662-3.
13. Gurvits GE, Cherian K, Shami MN, Korabathina R, El-Nader EM, Rayapudi K, et al. Black esophagus: new insights and multicenter international experience in 2014. Dig Dis Sci. 2015; 60(2):444-53.

14. Augusto F, Fernandes V, Cremers MI, Oliveira AP, Lobato C, Alves AL, et al. Acute necrotizing esophagitis: a large retrospective case series. Endoscopy. 2004; 36(5):411-5.

Trabalho recebido: $16 / 02 / 2018$

Trabalho aprovado: $11 / 07 / 2018$ 